\title{
Integrated Microheaters and the Role of Localized Temperature Control in Chemical and Biochemical Sensing
}

\author{
Steve Semancik \\ National Institute of Standards and Technology (NIST), Biomolecular Measurement Division \\ 100 Bureau Drive, Gaithersburg, Maryland, USA \\ stephen.semancik@nist.gov
}

\begin{abstract}
Temperature governs the nature of materials properties and varied interactional processes that can play a role in generating signals for chemical and biochemical sensing. Integrating heaters into microscale devices with other components required for sensing enables the control and measurement of temperature at the active portion of such devices. Fabrication methods and characteristics are described for two types of temperature-addressable platforms: microhotplate arrays of chemiresistive elements for gas-phase chemical sensing, and planar, 3-electrode electrochemical structures for stability measurements on solution-phase biochemicals. The manner by which rapid localized heating can enhance data streams for sensor-based chemical recognition, and also allow faster measurements of biomolecular stability of small-volume samples, is discussed.
\end{abstract}

Key words: chemical/biochemical sensing, chemiresistive, electrochemical, microheater, temperature

\section{Introduction}

Variations in thermal energy can activate/deactivate reactions, control adsorption and desorption kinetics, and also stimulate bond breaking, conformational changes and melting phenomena. For this reason, it can be advantageous to include localized temperature control in chemical and biochemical sensing devices as a means to collect (additional) information that is useful in identifying and characterizing the target species. Early conductometric gas sensor research demonstrated the benefits of operating different sensing materials at different fixed temperatures [1]. Later work showed that platforms capable of applying temperature gradients to sensing materials could amplify the information available for detection [2]. The development of integrated, low-power microheaters embedded within micromachined sensor structures created opportunities for having multiple sensing elements with individually-addressable and rapid temperature control [3]. Such monolithic arrays can have elements with different materials as well as different temperatures at any point in time. Temperatures of elements can also be varied or modulated as a function of time, and a great deal of analytical information could then be acquired from each array element in a relatively short time. Similarly, temperature programming or ramping can also be used in biosensing devices to monitor temperature-dependent interactions or measure melting curves. While temperature variation can be employed to advantage for a wide range of sensing principles, here platforms, concepts and methods developed at NIST are illustrated for temperature-programmed chemiresistive gas sensing, and for electrochemical monitoring of biomolecular conformation changes.

\section{Fabrication of Devices with Microheaters}

Depending on the type of sample and sensing principle being employed, the range of temperature variation required from a microheater will be different, as will the components that have to be compatibly integrated with the thermal control components. In general, the more robust materials involved in gas-phase chemical sensing can benefit with excursions from $20{ }^{\circ} \mathrm{C}$ to $\sim 500{ }^{\circ} \mathrm{C}$, while in biosensing one would not typically exceed maximum temperatures of $\sim 80{ }^{\circ} \mathrm{C}$. Lithographic methods have been used to realize multicomponent sensor structures by processes that are readily compatible with array fabrication [4]. The multilevel structures include 
polysilicon or $\mathrm{Pt}$ resistive heating elements, electrodes of differing materials, connecting traces and insulating layers. Temperature measurement makes use of calibrated resistance or power.

Figure 1a shows a single chemiresistive microhotplate gas sensor element, while Figure $1 \mathrm{~b}$ shows the type of array configuration that can include individually-addressable replicates of the single element. These devices were constructed on a silicon wafer and then portions of the underlying structure were micromachined (etched) in order to produce the thermallyisolated suspended structure operable from 20 ${ }^{\circ} \mathrm{C}$ to $480{ }^{\circ} \mathrm{C}$ with an efficiency of $\sim 25^{\circ} \mathrm{C} / \mathrm{mW}$. Top surface electrodes (interdigitated in Figure 1a) are used to probe electrical characteristics of over-deposited sensing materials. Thermal time constants for these elements range from 2 $\mathrm{ms}$ to $8 \mathrm{~ms}$ for heating and cooling [4].

Figure 2 shows a planar 3-electrode (WE, CE, $\mathrm{RE})$ electrochemical device developed for measuring melting temperatures of biomolecular species [5]. No micromachining is used for this device, which probes smallvolume, solution-phase samples, but the $\mathrm{SiO}_{2}$ insulating layer between the $\mathrm{Pt}$ heater and top electrodes must be thin for thermal transfer, but of high quality to avoid electrical leakage.

\section{Gas-Phase Chemical Sensing}

A prime advantage of the microheater devices (Figure 1) is the ability to perform higher dimensionality sensing. A single sensor can act like many virtual sensors as its temperature is rapidly varied in time; even more information can be quickly obtained from the independent temperature-programmed elements in an array. Systematic data acquisition has been used to

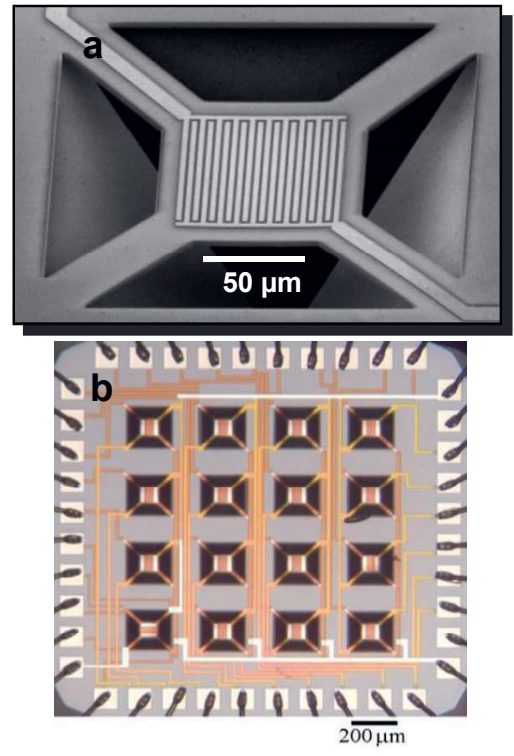

Fig. 1. a) Single microelement; b) 16-element array.

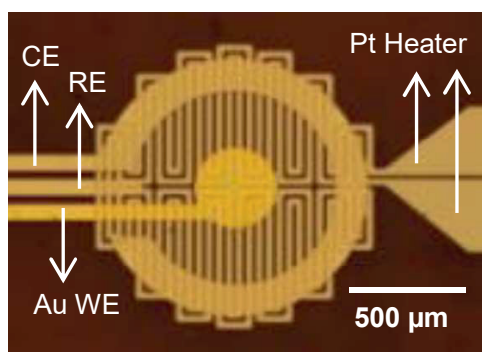

Fig. 2. Planar 3-electrode electrochemical platform. optimize temperature programs for applicationspecific sensing [6,7], and to do hierarchical recognition of gas species using prescribed portions of the programmed databases [8]. It is worth noting that the localized heating capability of these elements can also be employed for self-lithographic CVD deposition of sensing materials, efficient processing-performance studies, and to burn off protective coatings to activate fresh sensor elements.

\section{Biochemical Sensing}

To demonstrate the capabilities of the device in Figure 2, various forms of DNA (duplex, Gquadruplex) have been immobilized on the $\mathrm{Au}$ working electrode. Hybridization with a methylene blue-tagged DNA strand creates a tethered duplex structure whose electron transfer is monitored as the microheater is used to elevate sample temperature through/beyond the melting temperature, $\mathrm{T}_{\mathrm{m}}$ [5]. Differences in $\mathrm{T}_{\mathrm{m}}$ associated with single nucleotide polymorphism have been observed. Using local temperature ramps the stability of biomolecular forms, as a function of chemical environment and introduced ligand type, can be investigated with these low sample volume devices.

\section{Acknowledgments}

Numerous persons who contributed to the microsensor program at NIST over the years are cited in the references 3-8. I also acknowledge funding from DoE, NASA, DoD and the NIST-National Research Council Postdoctoral Associateship Program.

\section{References}

[1] e.g. - N. Yamazoe, N. Miura, Chemical Sensor Technology, Vol. 4, p.19-42 (1992).

[2] V. Sysoev, I. Kiselev, M. Frietsch, J. Goschnick, Sensors 4, 37-46 (2004).

[3] S. Semancik, R. Cavicchi, M. Gaitan, J. Suehle, US Patent 5345213A (1994).

[4] S. Semancik, R. Cavicchi, Accounts of Chemical Research 31, 279-287 (1998).

[5] Z. Shen, H. Sintim, S. Semancik, Analytica Chimica Acta 853, 265-270 (2015).

[6] T. Kunt, T. McAvoy, R. Cavicchi, S. Semancik, Sensors \& Actuators B53, 24-43 (1998).

[7] P. Rogers, S. Semancik, Sensors \& Actuators B163, 8-19 (2012).

[8] B. Raman, J. Hertz, K. Benkstein, S. Semancik, Analytical Chemistry 80, 8364-8371 (2008). 